(D) Adriana dos Santos Dutra ${ }^{1}$

(D) Gisselma Aliny Santos Muniz ${ }^{1}$

(1D) Antonia Caroline Diniz Brito ${ }^{2}$

(D) Andréa Martins Melo

Fontenele ${ }^{3}$

(D) Sueli Ismael Oliveira da

Conceição ${ }^{4}$

${ }^{1}$ Universidade Federal do Maranhão, Programa de Pós-

Graduação Residência

Multiprofissional em Saúde,

Hospital Universitário da

Universidade Federal do

Maranhão. São Luis, MA, Brasil.

2 Universidade Federal do Maranhão, Programa de Pós-

Graduação em Ciências da Saúde. São Luis, MA, Brasil.

3 Universidade Federal do Maranhão, Hospital Universitário da Universidade Federal do Maranhão, Serviço de Nefrologia. São Luis, MA, Brasil.

4 Universidade Federal do Maranhão, Centro de Ciências Biológicas e da Saúde,

Departamento de Ciências

Fisiológicas. São Luis, MA, Brasill.

Correspondência

Sueli Ismael Oliveira da Conceição sueli.ismael@gmail.com

\section{Avaliação do risco cardiovascular em pacientes renais crônicos em fase não dialítica por indicadores antropométricos}

\author{
Cardiovascular risk evaluation in chronic renal patients in \\ non-dialysis by anthropometric indicators
}

\section{Resumo}

Introdução: Estudos que adotaram os indicadores antropométricos de risco cardiovascular na avaliação de pacientes com doença renal crônica (DRC) em fase não dialítica são escassos. Objetivo: Avaliar o risco cardiovascular por indicadores antropométricos em pacientes com DRC em fase não dialítica e fatores associados. Métodos: Estudo transversal com 106 pacientes atendidos em hospital universitário, em São Luís-MA. Aplicou-se formulário estruturado com informações sociodemográficas, estilo de vida, morbidades e estadiamento da doença renal. Para avaliação antropométrica e identificação do risco cardiovascular, adotaram-se os indicadores: índice de massa corporal, circunferência da cintura, circunferência do pescoço, diâmetro abdominal sagital, relação cintura-estatura e índice de conicidade. Os testes do Qui-quadrado de Person e Exato de Fischer avaliaram a associação entre as variáveis e adotou-se o nível de significância $p<0,05$. Resultados: Pela circunferência da cintura, houve risco cardiovascular muito elevado nas mulheres $(75,4 \%)$, e pelo índice de conicidade, todas estavam em risco $(p<0,001)$. Nos pacientes com $\geq 60$ anos de idade, houve risco cardiovascular pelos indicadores diâmetro abdominal sagital $(77,5 \%)$, razão cintura-estatura $(92,6 \%)$ e índice de conicidade $(98,2 \%)(p<0,005)$. A circunferência do pescoço apontou risco elevado nos fumantes $(100,0 \%)$ e etilistas $(88,9 \%)(p=0,001)$. Pela circunferência do pescoço $(73,5 \%)$ e razão cintura-estatura $(91,7 \%)$, os renais crônicos diabéticos apresentaram risco cardiovascular elevado $(p<0,05)$. Conclusão: O risco cardiovascular evidenciado por diferentes indicadores antropométricos aponta que ações de promoção da saúde devem ser implementadas, de modo a melhorar o estilo de vida e contribuir para o melhor prognóstico desses pacientes.

Palavras-chave: Antropometria. Doenças Cardiovasculares. Insuficiência Renal Crônica.

\section{Abstract}

Introduction: Studies that have adopted anthropometric indicators for the evaluation of cardiovascular risk in patients with chronic kidney disease (CKD) in the non-dialysis phase are scarce. Objective: To assess cardiovascular risk, with anthropometric indicators, in patients with CKD in the non-dialysis phase and their associated factors. Methods: Cross-sectional study with 106 patients treated at a university hospital in São Luís-MA. A structured form was applied to collect information on sociodemographics, lifestyle, morbidities, and stage of kidney disease. For anthropometric evaluation and identification of cardiovascular risk, the following indicators were adopted: body mass index, waist circumference, neck circumference, sagittal abdominal diameter, waist-toheight ratio, and conicity index. Person's chi-square test and Fischer's exact test evaluated the association between the variables and adopted the significance level 


\section{DEMETRA}

$p<0.05$. Results: According to waist circumference, there was very high cardiovascular risk in women (75.4\%); and according to the conicity index, all were at risk $(p<0.001)$. In patients with $\geq 60$ years of age, there was cardiovascular risk according to the indicators of sagittal abdominal diameter (77.5\%), waist-to-height ratio (92.6\%), and conicity index (98.2\%) ( $p<0.005)$. Neck circumference showed high risk in smokers (100.0\%) and alcohol users (88.9\%) ( $p=0.001)$. According to neck circumference (73.5\%) and waist-to-height ratio (91.7\%), diabetic chronic renal patients presented high cardiovascular risk $(p<0.05)$. Conclusion: The cardiovascular risk, evidenced by different anthropometric indicators, shows that actions promoting a healthy lifestyle should be implemented, thus contributing to the better prognosis of these patients.

Keywords: Anthropometry. Cardiovascular diseases. Chronic Renal Insufficiency. 


\section{INTRODUÇÃO}

A doença renal crônica (DRC) compreende anormalidades da estrutura ou função renal, presentes por mais de três meses, com complicações para a saúde, e pode ser classificada em diferentes estágios, de acordo com o grau de redução da filtração glomerular. ${ }^{1}$

Em nível mundial, a prevalência da DRC é elevada e atinge de 10 a 15\% da população. ${ }^{2}$ Inquérito realizado no Brasil, em 2013, com indivíduos com $\geq 18$ anos de idade, mostrou que 1,4\% deles apresentam algum estágio de DRC. Na Região Nordeste, 1,2\% dos indivíduos possuem algum grau de disfunção renal, e no estado do Maranhão, a prevalência da DRC é de $0,8 \% .^{3}$

As doenças cardiovasculares (DCV) são consideradas uma das principais complicações da $\mathrm{DRC}^{4}$ e a principal causa de morte no mundo. ${ }^{5}$ Os fatores de risco para DCV em pacientes com DRC incluem os tradicionais (hipertensão, diabetes, dislipidemia, tabagismo, entre outros) e não tradicionais (inflamação, anemia, estresse oxidativo e distúrbios do metabolismo mineral).

A fim de contribuir para a redução do risco cardiovascular, que é potencializado pela presença da DRC nos pacientes, é fundamental realizar a avaliação do estado nutricional de forma criteriosa. ${ }^{6}$ Para isso, é necessário empregar diferentes métodos, de modo a se obter informações confiáveis. Dentre os métodos de avaliação nutricional, destaca-se a antropometria, por ser de fácil aplicação, baixo custo e muito utilizada na prática clinica. ${ }^{7}$

Os indicadores antropométricos de risco cardiovascular mais adotados na prática clínica são: circunferência da cintura (CC), ${ }^{7}$ razão cintura-estatura (RCE) ${ }^{8}$ e diâmetro abdominal sagital (DAS). ${ }^{9}$ Também vem sendo demonstrada forte correlação entre o índice de conicidade $(\mathrm{IC})^{10} \mathrm{e}$ circunferência do pescoço (CPesc) e fatores de risco cardiovascular. ${ }^{11}$

Considerando-se que a DRC e DCV são problemas de saúde pública a serem enfrentados no Brasil e que a maior parcela das pesquisas é conduzida em pacientes com DRC em fase dialítica, este estudo teve como objetivo avaliar o risco cardiovascular de indicadores antropométricos e os fatores associados em pacientes com DRC em fase não dialítica, atendidos em hospital universitário de referência em São Luís-MA.

\section{MÉTODOS}

\section{Protocolo e amostra do estudo}

Estudo transversal integrado à pesquisa "Inflamação e risco cardiovascular em pacientes renais crônicos não dialíticos", desenvolvido em um Centro de Referência em Doenças Renais de um Hospital Universitário, localizado em São Luís, Maranhão, no período de maio a agosto de 2017.

A amostra deste estudo foi não probabilística e constituída por pacientes com DRC em tratamento não dialítico, atendidos no ambulatório do hospital de referência. Incluíram-se indivíduos de ambos os sexos, adultos e idosos e que aceitaram participar da pesquisa. Não foram incluídos gestantes e pacientes com algum membro amputado, apenas um rim, registro de internação hospitalar no mês anterior, história de diálise anterior, insuficiência hepática, diabetes tipo I, sinais clínicos de inflamação aguda, instáveis clinicamente (sintomas urêmicos), 


\section{DEMETRA}

doenças infectocontagiosas, doenças crônicas consumptivas (câncer, insuficiência cardíaca grave e síndrome da imunodeficiência adquirida) e em uso de imunossupressores. A amostra final resultou em 106 pacientes.

\section{Coleta de dados}

Uma equipe treinada realizou as entrevistas e aplicou aos pacientes formulários estruturados para a coleta de informações: demográficas (sexo: masculino e feminino; idade em anos: 20-59 ou ₹60; cor da pele autorreferida: branca, preta ou parda e outras; e o estado civil: solteiro, casado/união consensual, viúvo e separado); socioeconômicas (escolaridade: analfabeto/fundamental incompleto, fundamental । completo/fundamental \| incompleto, fundamental ॥ completo/médio incompleto, médio completo/superior incompleto e superior completo/pós-graduado; total de moradores no domićlio: até 4 e >4; renda familiar em salários mínimos: $>1, \geq 1$ a $\leq 2,>2$ a $<4$ e $\geq 4$ ); estilo de vida (hábito de fumar: sim, não e parou; e ingestão de bebidas alcóolicas: sim, não e parou).

Obtiveram-se as seguintes informações dos prontuários: presença de morbidades (hipertensão arterial: sim e não; e diabetes mellitus tipo ll: sim e não) e estadiamento da DRC, classificando-o com base em referência específica. ${ }^{1}$ A fórmula do CKD-EPI ( $\left.\mathrm{ml} / \mathrm{min} / 1,73^{2}\right)$ foi utilizada na estimativa da taxa de filtração glomerular (eTFG). ${ }^{12}$

As medidas antropométricas dos pacientes foram aferidas por meio de equipamentos e técnicas padronizadas na literatura. Com base no peso corporal e na altura, calculou-se o índice de massa corporal (IMC). Na classificação do IMC (kg/m²), adotaram-se os pontos de cortes da Organização Mundial da Saúde $(\mathrm{OMS}){ }_{1}^{13}$ categorizados em magreza, eutrofia e excesso de peso (sobrepeso e obesidade).

As medidas da CC foram categorizadas para ambos os sexos em: sem risco, risco elevado e risco muito elevado, de acordo com os critérios de referência. ${ }^{14}$ Para a Cpesc, considerou-se em risco para excesso de peso quando a medida resultou em $>37 \mathrm{~cm}$ nos homens e $>34 \mathrm{~cm}$ nas mulheres. ${ }^{11}$ Para as medidas do DAS, considerou-se como indicativo de risco cardiovascular quando $\geq 20,5 \mathrm{~cm}$ para homens e $\geq 19,5 \mathrm{~cm}$ para mulheres. ${ }^{9}$

A RCE foi determinada pela razão da CC pela estatura dos pacientes e adotaram-se os pontos de corte $\geq 0,52$ para homens e $\geq 0,53$ para mulheres como indicativo de risco cardiovascular. ${ }^{8} \mathrm{O}$ IC foi calculado conforme estabelecido por Valdez, ${ }^{15}$ utilizando como pontos de corte para o risco cardiovascular: $\geq 1,25$ para homens e $\geq 1,18$ para mulheres. ${ }^{10}$

Cpesc, DAS, RCE e IC foram categorizados para ambos os sexos em: sem risco e com risco.

\section{Análise estatística}

As análises estatísticas foram conduzidas no software Stata®, versão 14.0. A normalidade das variáveis contínuas foi verificada por meio do teste de Shapiro Wilk. As variáveis contínuas foram apresentadas por meio de média e desvio padrão, e as variáveis categóricas, por meio de frequências absolutas e relativas. Os testes do Qui-quadrado de Person e Exato de Fischer foram aplicados para verificar a associação entre as variavéis categóricas. Considerou-se valor de $p<0,05$ como estatisticamente significativa. 


\section{Aspectos éticos}

O estudo foi aprovado pelo Comitê de Ética do Hospital Universitário da Universidade Federal do Maranhão sob parecer consubstanciado n 2.015.866/2017 e atendeu à Resolução n 466/2012 do Conselho Nacional de Saúde. Todos os participantes assinaram o Termo de Consentimento Livre e Esclarecido.

\section{RESULTADOS}

Dentre os pacientes avaliados, predominou o sexo feminino (53,8\%); a idade média da amostra foi 59,2 (Dp $\pm 13,6$ ) anos. A maior frequência dos pacientes referiu ter cor da pele parda ou outras (71,7\%), era casada ou mantinha união estável (66,0\%), residia com até quatro moradores no domicílio (67,9\%), possuía renda familiar de um a dois salários-mínimos $(56,6 \%)$ e não era fumante $(58,4 \%)$. Elevada parcela dos investigados estava nos estágios 1 e 2 da DRC (45,2\%), tinha hipertensão arterial (95,2\%) e diabetes mellitus (53,3\%). A maior frequência das mulheres cursou o Ensino Fundamental I completo/Fundamental II incompleto (42,9\%) e maior proporção dos homens tinha o Ensino Médio Completo/superior incompleto $(38,8 \%)(p=0,023)$. Dentre os pacientes que não consumiam bebida alcóolica, predominaram as mulheres $(66,8 \%)(p=0,039)$ (Tabela 1).

Tabela 1. Características sociodemográficas, estadiamento da doença, morbidades e estilo de vida de pacientes renais crônicos em fase não dialítica, segundo o gênero. São Luís-MA, 2017.

\begin{tabular}{|c|c|c|c|c|}
\hline Variáveis & $\begin{array}{l}\text { Total } \\
\text { n (\%) }\end{array}$ & $\begin{array}{l}\text { Homem } \\
\mathrm{n}(\%)\end{array}$ & $\begin{array}{l}\text { Mulher } \\
\mathrm{n}(\%)\end{array}$ & $p$ \\
\hline \multirow[t]{2}{*}{ Sexo } & & & & - \\
\hline & $106(100)$ & $49(46,2)$ & $57(53,8)$ & \\
\hline Idade (anos) & & & & $0,869 * *$ \\
\hline $29-59$ & $51(48,1)$ & $24(49,0)$ & $27(47,4)$ & \\
\hline$\geq 60$ & $55(51,9)$ & $25(51,0)$ & $30(52,6)$ & \\
\hline Cor da pele & & & & $0,678^{* *}$ \\
\hline Branca & $14(13,2)$ & $6(12,2)$ & $8(14,0)$ & \\
\hline Preta & $16(15,1)$ & $9(18,4)$ & $7(12,3)$ & \\
\hline Parda ou outras & $76(71,7)$ & $34(69,4)$ & $42(73,7)$ & \\
\hline Estado civil & & & & $0,253^{* * *}$ \\
\hline Solteiro & $14(13,2)$ & $7(14,3)$ & $7(12,3)$ & \\
\hline Casado/União estável & $70(66,0)$ & $34(69,3)$ & $36(63,1)$ & \\
\hline Viúvo & $16(15,1)$ & $4(8,2)$ & $12(21,1)$ & \\
\hline Separado & $6(5,7)$ & $4(8,2)$ & $2(3,5)$ & \\
\hline Escolaridade* & & & & $0,023 * * *$ \\
\hline Analfabeto/ Fundamental I incompleto & $24(22,9)$ & $9(18,4)$ & $15(26,8)$ & \\
\hline Fundamental I completo/ Fundamental II incompleto & $34(32,4)$ & $10(20,4)$ & $24(42,9)$ & \\
\hline Fundamental II completo/ Médio incompleto & $8(7,6)$ & $6(12,2)$ & $2(3,6)$ & \\
\hline Médio completo/ Superior incompleto & $32(30,5)$ & $19(38,8)$ & $13(23,1)$ & \\
\hline Superior completo/ Pós-graduação & $7(6,6)$ & $5(10,2)$ & $2(3,6)$ & \\
\hline Total de moradores no domicilio & & & & $0,906^{* \star}$ \\
\hline Até 4 & $72(67,9)$ & $33(67,3)$ & $39(68,4)$ & \\
\hline Mais que 4 & $34(32,1)$ & $16(32,7)$ & $18(31,6)$ & \\
\hline
\end{tabular}


Tabela 1. Características sociodemográficas, estadiamento da doença, morbidades e estilo de vida de pacientes renais crônicos em fase não dialítica, segundo o gênero. São Luís-

$$
\text { MA, 2017. (Cont.) }
$$

\begin{tabular}{|c|c|c|c|c|}
\hline Variáveis & $\begin{array}{l}\text { Total } \\
\text { n (\%) }\end{array}$ & $\begin{array}{l}\text { Homem } \\
\mathrm{n}(\%)\end{array}$ & $\begin{array}{l}\text { Mulher } \\
\mathrm{n}(\%)\end{array}$ & $p$ \\
\hline Renda familiar (salários-mínimos) & & & & $0,617^{* \star *}$ \\
\hline$<1$ & $9(8,4)$ & $4(8,2)$ & $5(8,8)$ & \\
\hline 1 a 2 & $60(56,6)$ & $25(51,0)$ & $35(61,3)$ & \\
\hline$>2$ e $<4$ & $24(22,6)$ & $12(24,5)$ & $12(21,1)$ & \\
\hline 4 ou mais & $13(12,3)$ & $8(16,3)$ & $5(8,8)$ & \\
\hline Estadiamento da doença & & & & $0,190 * \star \star$ \\
\hline Estágio 1 & $24(22,6)$ & $8(16,3)$ & $16(28,1)$ & \\
\hline Estágio 2 & $24(22,6)$ & $14(28,6)$ & $10(17,5)$ & \\
\hline Estágio $3^{a}$ & $21(19,8)$ & $7(14,3)$ & $14(24,6)$ & \\
\hline Estágio 3b & $21(19,8)$ & $13(26,5)$ & $8(14,0)$ & \\
\hline Estágio 4 & $13(12,3)$ & $5(10,2)$ & $8(14,0)$ & \\
\hline Estágio 5 & $3(2,8)$ & $2(4,1)$ & $1(1,8)$ & \\
\hline \multicolumn{5}{|l|}{ Morbidades } \\
\hline Hipertensão arterial sistêmica* & & & & $>0,999 * * *$ \\
\hline Não & $5(4,8)$ & $2(4,1)$ & $3(5,4)$ & \\
\hline Sim & $100(95,2)$ & $47(95,9)$ & $53(94,6)$ & \\
\hline Diabetes mellitus* & & & & $0,958^{* *}$ \\
\hline Não & $56(53,3)$ & $26(53,1)$ & $30(53,6)$ & \\
\hline $\operatorname{Sim}$ & $49(46,7)$ & $23(46,9)$ & $26(46,4)$ & \\
\hline Hábito de fumar & & & & $0,290 * \star *$ \\
\hline Não & $62(58,4)$ & $25(51,0)$ & $37(64,9)$ & \\
\hline Sim & $2(1,8)$ & $1(2,0)$ & $1(1,8)$ & \\
\hline Parou & $42(39,6)$ & $23(47,0)$ & $19(33,3)$ & \\
\hline Ingestão de bebida alcoólica & & & & $0,039 * * *$ \\
\hline Não & $60(56,6)$ & $22(44,9)$ & $38(66,8)$ & \\
\hline $\operatorname{sim}$ & $9(8,5)$ & $7(14,3)$ & $2(3,5)$ & \\
\hline Parou & $37(34,9)$ & $20(40,8)$ & $17(29,7)$ & \\
\hline TOTAL & $106(100)$ & $49(100)$ & $57(100)$ & \\
\hline
\end{tabular}

*Variação da amostra decorrente de eventual perda de informação.

**Teste qui-quadrado de Pearson

$* * *$ Teste Exato de Fischer

A avaliação antropométrica por meio do IMC evidenciou que o excesso de peso prevaleceu nos pacientes com 29-59 anos de idade $(66,7 \%)(p=0,023)$. Pela CC, o risco elevado para desenvolver DCV foi maior nos homens $(34,7 \%)$ e o risco muito elevado predominou nas mulheres $(75,4 \%)(p<0,001)$. Pelo IC, todas as mulheres apresentaram risco de desenvolver DCV $(p<0,001)$. Segundo o DAS (77,5\%), RCE $(92,6 \%)$ e IC $(98,2 \%)$, os pacientes com $\geq 60$ anos de idade apresentaram maior risco de DCV que aqueles com 29-59 anos ( $p<0,05)$. Não houve diferença estatística significativa entre os indicadores IMC, Cpesc, DAS e RCE e o sexo, assim como entre CC e Cpesc e a idade ( $p>0,05)$ (Tabela 2). 
Tabela 2. Índice de massa corporal e indicadores antropométricos de risco cardiovascular, segundo o sexo e a idade dos pacientes renais crônicos em fase não dialítica.

São Luís-MA, 2017.

\begin{tabular}{|c|c|c|c|c|c|c|c|}
\hline \multirow[b]{2}{*}{ Variáveis } & \multirow[b]{2}{*}{$\begin{array}{l}\text { Total } \\
\text { n (\%) }\end{array}$} & \multicolumn{2}{|l|}{ Sexo } & \multirow[b]{2}{*}{$p$} & \multicolumn{2}{|c|}{ Idade (anos) } & \multirow[b]{2}{*}{$p$} \\
\hline & & $\begin{array}{l}\text { Homem } \\
\%\end{array}$ & $\begin{array}{l}\text { Mulher } \\
\%\end{array}$ & & $\begin{array}{l}29 \text { a } 59 \\
\%\end{array}$ & $\begin{array}{l}\geq 60 \\
\%\end{array}$ & \\
\hline ÍMC $(n=106)$ & & & & $0,528 * * *$ & & & $0,023 * * *$ \\
\hline Magreza & $6(5,6)$ & 8,2 & 3,5 & & 0,0 & 10,9 & \\
\hline Eutrofia & $39(36,8)$ & 38,8 & 35,1 & & 33,3 & 40,0 & \\
\hline Excesso de peso & $61(57,6)$ & 53,1 & 61,4 & & 66,7 & 49,1 & \\
\hline$C C(n=106)$ & & & & $<0,001 * * *$ & & & $0,177^{* *}$ \\
\hline Sem risco & $23(21,7)$ & 38,8 & 7,0 & & 29,4 & 14,5 & \\
\hline Risco elevado & $27(25,5)$ & 34,7 & 17,6 & & 23,5 & 27,3 & \\
\hline Risco muito elevado & $56(56,0)$ & 26,5 & 75,4 & & 47,1 & 58,2 & \\
\hline CPesc $(n=105)^{\star}$ & & & & $0,523 * *$ & & & $0,151^{\star *}$ \\
\hline Sem risco & $42(40,0)$ & 36,7 & 42,9 & & 47,1 & 33,3 & \\
\hline Com risco & $63(60,0)$ & 63,3 & 57,1 & & 52,9 & 66,7 & \\
\hline DAS $(n=95)^{*}$ & & & & $0,212^{* *}$ & & & $0,001 * *$ \\
\hline Sem risco & $36(37,9)$ & 44,4 & 32,0 & & 54,3 & 22,5 & \\
\hline Com risco & $59(62,1)$ & 55,6 & 68,0 & & 45,7 & 77,5 & \\
\hline $\operatorname{RCE}(n=105)^{*}$ & & & & $0,565^{* *}$ & & & $0,005 * * *$ \\
\hline Sem risco & $19(18,1)$ & 20,4 & 16,1 & & 29,4 & 7,4 & \\
\hline Com risco & $86(81,9)$ & 79,6 & 83,9 & & 70,6 & 92,6 & \\
\hline íc $(n=105)^{*}$ & & & & $<0,001 * * *$ & & & $0,007 * * *$ \\
\hline Sem risco & $10(9,5)$ & 20,4 & 0,0 & & 17,6 & 1,8 & \\
\hline Com risco & $95(90,5)$ & 79,6 & 100,0 & & 82,4 & 98,2 & \\
\hline
\end{tabular}

*Variação da amostra decorrente de eventual perda de informação.

IMC: índice de massa corporal; CC: circunferência da cintura; CPesc: circunferência do pescoço; DAS: diâmetro abdominal sagital; RCE: razão cintura-estatura; IC:índice de conicidade. **Teste qui-quadrado de Pearson

$* * *$ Teste Exato de Fischer

Avaliando o indicador Cpesc, verificou-se que todos os entrevistados que eram fumantes e 78,6\% daqueles que pararam de fumar apresentaram maior risco de desenvolver DCV em comparação aos não fumantes ( $p=0,001)$. Também pela Cpesc, os pacientes etilistas $(88,9 \%)$ e os ex-etilistas $(86,1 \%)$ tiveram maior risco de apresentar DCV em comparação aos não etilistas $(p<0,001)$. Não houve diferença estatística significativa entre CC, DAS, RCE e IC com as variáveis hábito de fumar e entre CC, DAS, RCE e IC com a ingestão de bebida alcoólica $(p>0,05)($ Tabela 3). 
Tabela 3. Indicadores antropométricos de risco cardiovascular, segundo o estilo de vida dos pacientes renais crônicos em fase não dialítica. São Luís-MA, 2017.

\begin{tabular}{|c|c|c|c|c|c|c|c|c|c|}
\hline \multirow{2}{*}{ Variáveis } & \multirow[b]{2}{*}{$\begin{array}{l}\text { Total } \\
\text { n (\%) }\end{array}$} & \multicolumn{3}{|c|}{ Hábito de fumar } & \multicolumn{4}{|c|}{ Ingestão de bebida alcoólica } & \multirow{2}{*}{$p$} \\
\hline & & $\begin{array}{l}\text { Não } \\
\%\end{array}$ & $\begin{array}{l}\text { Sim } \\
\%\end{array}$ & $\begin{array}{l}\text { Parou } \\
\%\end{array}$ & $P$ & $\begin{array}{l}\text { Não } \\
\%\end{array}$ & $\begin{array}{l}\text { Sim } \\
\%\end{array}$ & $\begin{array}{l}\text { Parou } \\
\%\end{array}$ & \\
\hline$C C(n=106)$ & & & & & $0,516 * * *$ & & & & $0,691 * * *$ \\
\hline Sem risco & $23(21,7)$ & 24,2 & 0,0 & 19,1 & & 26,6 & 11,1 & 16,2 & \\
\hline Risco elevado & $27(25,5)$ & 21,0 & 0,0 & 33,3 & & 25,0 & 33,3 & 24,3 & \\
\hline Risco muito elevado & $56(56,0)$ & 54,8 & 100,0 & 47,6 & & 48,3 & 55,6 & 59,5 & \\
\hline Cpesc $(n=105)^{\star}$ & & & & & $0,001 * * *$ & & & & $<0,001 * * *$ \\
\hline Sem risco & $42(40,0)$ & 54,1 & 0,0 & 21,4 & & 60,0 & 11,1 & 13,9 & \\
\hline Com risco & $63(60,0)$ & 45,9 & 100,0 & 78,6 & & 40,0 & 88,9 & 86,1 & \\
\hline DAS $(n=95) *$ & & & & & $0,227 * * *$ & & & & $0,069 * * *$ \\
\hline Sem risco & $36(37,9)$ & 44,6 & 0,0 & 29,7 & & 47,2 & 11,1 & 30,3 & \\
\hline Com risco & $59(62,1)$ & 55,4 & 100,0 & 70,3 & & 52,8 & 88,9 & 69,7 & \\
\hline $\operatorname{RCE}(n=105)^{*}$ & & & & & $0,345 * * *$ & & & & $0,173 * * *$ \\
\hline Sem risco & $19(18,1)$ & 22,9 & 0,0 & 11,9 & & 23,7 & 0,0 & 13,5 & \\
\hline Com risco & $86(81,9)$ & 77,1 & 100,0 & 88,1 & & 76,3 & 100,0 & 86,5 & \\
\hline ÍC (n=105)* & & & & & $>0,999 * * *$ & & & & $0,684 * * *$ \\
\hline Sem risco & $10(9,5)$ & 9,8 & 0,0 & 9,5 & & 11,8 & 0,0 & 8,1 & \\
\hline Com risco & $95(90,5)$ & 90,2 & 100,0 & 90,5 & & 88,2 & 100,0 & 91,9 & \\
\hline
\end{tabular}

* Variação da amostra decorrente de eventual perda de informação.

CC: circunferência da cintura; CPesc: circunferência do pescoço; DAS: diâmetro abdominal sagital; RCE: razão cintura-estatura; IC:índice de conicidade.

**Teste qui-quadrado de Pearson

$\star \star \star T e s t e$ Exato de Fischer 
A análise da tabela 4 mostra que, segundo a Cpesc (73,5\%) e RCE (91,7\%), os pacientes com DRC diabéticos apresentaram maior risco de DCV quando comparados aos com DRC não diabéticos $(p<0,05)$. Não houve significância estatística entre CC, DAS, IC e a variável diabetes mellitus tipo II, assim como entre todos os indicadores antropométricos e a hipertensão arterial $(p>0,05)$

Tabela 4. Indicadores antropométricos de risco cardiovascular, segundo as comorbidades dos pacientes renais crônicos em fase não dialítica. São Luís-MA, 2017.

\begin{tabular}{|c|c|c|c|c|c|c|c|}
\hline \multirow[b]{2}{*}{ Variáveis } & \multirow[b]{2}{*}{$\begin{array}{l}\text { Total } \\
\text { n (\%) }\end{array}$} & \multicolumn{2}{|c|}{ Hipertensão arterial } & \multirow[b]{2}{*}{$p$} & \multicolumn{2}{|c|}{ Diabetes mellitus } & \multirow[b]{2}{*}{$p$} \\
\hline & & $\begin{array}{l}\text { Não } \\
\%\end{array}$ & $\begin{array}{l}\text { Sim } \\
\%\end{array}$ & & $\begin{array}{l}\text { Não } \\
\%\end{array}$ & $\begin{array}{l}\text { Sim } \\
\%\end{array}$ & \\
\hline$C C(n=106)$ & & & & $\begin{array}{l}0,707 * \\
\star *\end{array}$ & & & $\begin{array}{l}0,195 * \\
* *\end{array}$ \\
\hline Sem risco & $23(21,7)$ & 40,0 & 21,0 & & 28,6 & 14,3 & \\
\hline Risco elevado & $27(25,5)$ & 20,0 & 26,0 & & 25,0 & 26,5 & \\
\hline Risco muito elevado & $56(56,0)$ & 40,0 & 53,0 & & 46,4 & 59,2 & \\
\hline Cpesc $(n=105)^{\star}$ & & & & $\begin{array}{l}0,155^{*} \\
\star *\end{array}$ & & & $\begin{array}{l}0,007 * \\
\star\end{array}$ \\
\hline Sem risco & $42(40,0)$ & 80,0 & 38,4 & & 52,7 & 26,5 & \\
\hline Com risco & $63(60,0)$ & 20,0 & 61,6 & & 47,3 & 73,5 & \\
\hline $\operatorname{DAS}(n=95)^{*}$ & & & & $\begin{array}{l}0,066^{*} \\
\star *\end{array}$ & & & $\begin{array}{l}0,061 * \\
\star\end{array}$ \\
\hline Sem risco & $36(37,9)$ & 80,0 & 35,6 & & 46,9 & 28,3 & \\
\hline Com risco & $59(62,1)$ & 20,0 & 64,4 & & 53,1 & 71,7 & \\
\hline $\operatorname{RCE}(n=105)^{\star}$ & & & & $\begin{array}{l}0,225^{*} \\
\star *\end{array}$ & & & $\begin{array}{l}0,015 * \\
\star\end{array}$ \\
\hline Sem risco & $19(18,1)$ & 40,0 & 17,2 & & 26,8 & 8,3 & \\
\hline Com risco & $86(81,9)$ & 60,0 & 82,8 & & 73,2 & 91,7 & \\
\hline ÍC $(n=105)^{\star}$ & & & & $\begin{array}{l}0,403 * \\
* *\end{array}$ & & & $\begin{array}{l}0,335 * \\
\star *\end{array}$ \\
\hline Sem risco & $10(9,5)$ & 20,0 & 9,1 & & 12,5 & 6,3 & \\
\hline Com risco & $95(90,5)$ & 80,0 & 90,9 & & 87,5 & 93,7 & \\
\hline
\end{tabular}

*Variação da amostra decorrente de eventual perda de informação.

CC: circunferência da cintura; CPesc: circunferência do pescoço; DAS: diâmetro abdominal sagital; RCE: razão cinturaestatura; IC:índice de conicidade.

**Teste qui-quadrado de Pearson

$* * *$ Teste de Exato de Fischer

\section{DISCUSSÃO}

Neste estudo, segundo os indicadores antropométricos CC e IC, o risco de DCV em pacientes com DRC em fase não dialítica se associou com o sexo. O risco de DCV pelo DAS, RCE e IC se associou com a idade. Pela Cpesc, houve risco de DCV mais elevado entre os pacientes DRC fumantes e os ex-fumantes, assim como entre os etilistas e os ex-etilistas. Pela Cpesc e a RCE, ser DRC diabético aumentou o risco de DCV.

Elevado número de pacientes DRC adultos estava com excesso de peso e a magreza predominou nos idosos. Estudos realizados no Brasil em $2013^{16}$ e $2019^{17}$ revelam que mais da metadade da população está 
com excesso de peso (56,9\% vs 55,4\%). Devido a sua magnitude na população, ${ }^{18}$ o sobrepeso e a obesidade representam um problema de saúde pública no país e são fatores de risco para o desenvolvimento das doenças crônicas não-transmissíveis. ${ }^{3}$

Ressalta-se que a prevalência de excesso de peso nos pacientes avaliados foi mais elevada que a estimada para a população brasileira adulta. ${ }^{17}$ Entretanto, a magreza percebida nos pacientes idosos foi de 10,9\%, contrariando os resultados de estudos nacionais que apontam o predomínio do excesso de peso nesse grupo, quando comparado ao baixo peso. ${ }^{17,19}$ É possível que a maior vulnerabilidade dos idosos, juntamente com alterações metabólicas, hormonais e inflamatórias, frequentemente observadas nos pacientes com DRC, ${ }^{20}$ contribuíram para o desencadeamento da magreza no grupo avaliado.

A literatura científica mostra que o excesso de peso em pacientes com DRC pode conferir proteção e não morbidade, implicando a evidência da epidemiologia reversa da obesidade. ${ }^{21}$ No entanto, há registos de que a massa magra corporal seja o melhor preditor de risco de mortalidade nos pacientes com DRC, ${ }^{22}$ pois eles podem apresentar valores distorcidos de IMC elevados, decorrentes da retenção hídrica provocada pela doença. Considerando que neste estudo não se avaliou a massa magra dos pacientes, o IMC apresenta limitações e deve ser avaliado com cautela, por não diferenciar a composição corporal. ${ }^{7}$ Logo, outros indicadores antropométricos devem ser considerados para avaliar o estado nutricional e o risco cardiovascular nesses pacientes.

Nesta investigação, o risco de DCV pela CC foi elevado nos homens e muito elevado nas mulheres com DRC. Contrariando estes achados, em pesquisa conduzida com 210 indivíduos hipertensos, com $\geq 18$ anos de idade, em São Luís-MA, observou-se que, segundo a CC, o risco muito elevado de DCV foi maior nos homens $(58,0 \%)$ em comparação com as mulheres $(56,0 \%) .{ }^{23}$ Cabe destacar que a CC é um indicador de adiposidade visceral e subcutânea e pode indicar predisposição ao diabetes mellitus e às DCV, tanto para as mulheres quanto para os homens deste estudo.

O IC é um indicador específico para avaliar risco DCV por estar fortemente associado com as mudanças de concentrações lipídicas, glicêmicas e pressão arterial dos indivíduos. Também indica que a obesidade central está mais associada ao risco cardiovascular do que a obesidade generalizada. ${ }^{10}$ Desse modo, segundo o IC, todas as mulheres desta investigação apresentaram risco de DCV, o que contesta os resultados do estudo que avaliou 109 indivíduos com mais de 20 anos de idade, em Recife-PE e verificou, pelo IC, que os homens $(X=1.3 \pm 0.1)$ apresentaram maior risco de DCV em comparação com as mulheres $(X=1.2 \pm 0.1){ }^{24}$

O crescente acúmulo de gordura na região abdominal e do tronco dos indivíduos predispõe a uma maior exposição aos eventos cardiovasculares ${ }^{13}$ e está relacionado às mudanças do estilo de vida ocorridas nas últimas décadas no Brasil, principalmente pela inadequação dos hábitos alimentares e pelo sedentarismo. ${ }^{16}$ É reconhecido que os alimentos ultraprocessados, por serem ricos em açúcares, gorduras e terem alta densidade calórica, também contribuem para desenvolvimento do excesso de peso corporal. ${ }^{25}$ Portanto, merece advertir que a adesão às melhores escolhas alimentares, prática de atividade física e cessação do tabagismo, dentre outras medidas, são necessárias para a mudança desse cenário.

Avaliando a idade dos pacientes, o risco de DCV pelos IC e RCE predominou entre os idosos. Esse resultado se assemelhou à investigação com 191 adultos e idosos de Salvador-BA, ao identificar predomínio de médias de IC e RCE mais elevadas em indivíduos com $\geq 60$ anos. ${ }^{26}$ Assim, ambos os indicadores possibilitam verificar mudanças na distribuição da gordura corporal entre indivíduos que tiveram diferentes medidas de peso corporal e/ou estatura, sendo bons disciminadores para a obesidade visceral. ${ }^{26}$

O DAS avalia a gordura visceral e se correlaciona fortemente com o risco cardiovascular. ${ }^{10} \mathrm{Um}$ estudo com 6.123 adultos e idosos finlandeses mostrou tendência para o aumento do DAS com o avanço da idade 
em ambos os sexos. O DAS médio foi maior nos indivíduos $\geq 50$ anos, em comparação com aqueles entre 30-49 anos, tanto para homens (22,4 [20,5-24,6] vs. 20,8 [19,3-22,7]), quanto para mulheres $(21,7[19,6-23,9]$ vs. $19,4[17,8-21,4]) .{ }^{27}$ De forma semelhante, com base no DAS, os idosos desta pequisa tiveram maior risco de DCV do que os adultos.

Vale ressaltar que o envelhecimento altera a composição corporal dos indivíduos, refletindo na redução de massa magra e elevação de massa gorda. ${ }^{28}$ Concordando com esta proposição, pesquisas nacionais mostram que a prevalência de excesso de peso na população idosa é maior que a de baixo peso, e a inadequação do padrão alimentar e o sedentarismo são alguns dos determinantes dessa condição, ${ }^{17,19}$ o que contribui para o maior risco cardiovascular nesse estágio da vida.

A Cpesc é utilizada como triagem para detecção de indivíduos com excesso de peso e está associada com risco de DCV. ${ }^{11}$ Neste estudo, a Cpesc mostrou risco para DCV nos pacientes com DRC tabagistas e nos ex-tabagistas, assim como nos etilistas e nos ex-etilistas.

Estudo realizado nas capitais brasileiras e Distrito Federal, em 2019, mostra que a estimativa do tabagismo em indivíduos com $\geq 18$ anos de idade se reduziu de 15,7\% para 9,8\%, nos últimos 13 anos, ${ }^{17}$ em consequência da implementação de ações educativas, preventivas, terapêuticas e regulatórias exitosas no país. ${ }^{29}$ Apesar dos avanços, deve-se considerar que o tabagismo é fator de risco cardiovascular para indivíduos renais crônicos, devendo, assim, ser abolido. Ademais, o hábito de fumar associado a obesidade, hipertensão arterial, dislipidemias e diabetes mellitus favorece o dano glomerular e reduz a função renal. ${ }^{4}$ Portanto, a condição do grupo investigado que é tabagista é preocupante e precisa ser revertida, pois o principal objetivo da terapêutica da fase não dialítica da DRC é retardar ao máximo a progressão da lesão renal.

De acordo com a OMS, o álcool é a droga mais consumida no mundo e 7,5\% da população mundial com idade $\geq 15$ anos ingerem essa substância. ${ }^{30}$ No Brasil, estima-se que 18,8\% da população adulta consomem bebida alcoólica abusivamente. ${ }^{17} \mathrm{O}$ consumo elevado de bebida alcoólica pode ser veículo para a maior ingestão de energia e está associado ao excesso de peso. ${ }^{31}$ Ademais, o etilismo pode aumentar a pressão arterial e o risco cardiovascular, potencializando, assim, a progressão da doença renal ${ }^{32}$ e a piora do quadro clínico dos pacientes com DRC. Logo, o etilismo deve ser desestimulado no grupo avaliado que referiu esse hábito.

Os pacientes com DRC diabéticos investigados apresentaram risco de DCV, segundo a Cpesc, o DAS e a RCE. O diabetes mellitus é um dos principais contribuintes para a progressão da doença renal dialítica, ${ }^{33} \mathrm{e}$ seu avanço desencadeia alterações macrovasculares, o que aumenta o risco de eventos cardiovasculares. ${ }^{34}$ Desse modo, os pacientes estão em condição mais vulnerável por apresentarem maior suscetibilidade à evolução da DRC e ao maior risco de mortalidade.

Este estudo apresentou algumas limitações, como a inexistência de pontos de corte específicos dos indicadores CC, Cpesc, DAS, RCE e IC para os idosos, o que impediu de verificar as diferenças de risco de DCV entre os indivíduos nos diferentes estágios da vida. Outra limitação refere-se ao fato de a amostra não ser probabilistica, o que impossibilitou fazer inferências para a população. Entretanto, como aspecto positivo, pode-se relatar que esta investigação foi pioneira em contribuir para o conhecimento do risco de DCV em pacientes com DRC em fase não dialítica, por meio dos indicadores antropométricos Cpesc, DAS, RCE e IC. 


\section{CONCLUSÕES}

Os pacientes com DRC em fase não dialítica apresentaram risco de DCV pelos indicadores antropométricos utilizados e que se associaram com o sexo, idade, estilo de vida e a presença de diabetes mellitus tipo II. Possivelmente, mudanças representadas pelo abandono do consumo do álcool e tabaco, além do controle do diabetes, poderão contribuir para o melhor prognóstico da DRC nesses pacientes.

A utilização continuada dos diferentes indicadores antropométricos de risco cardiovascular é relevante na prática clínica, uma vez que poderá subsidiar a orientação dos profissionais na implementação de ações de promoção da saúde e de enfrentamento da DRC não dialítica.

\section{AGRADECIMENTOS}

À Fundação de Amparo à Pesquisa e ao Desenvolvimento Tecnológico do Maranhão e aos pacientes que participaram do estudo.

\section{REFERÊNCIAS}

1. Kidney Diseases Improving Global Outcomes. Clinical practice guideline for the evaluation and management of Chronic Kidney Disease."Chapter 1: Definition and classification of CKD". Kidney Int. 2012; 3(1): 19-62.

2. Levin A, Tonelli M, Bonventre J, Coresh J, Donner J, Fogo AB. Global kidney health 2017 and beyond: a roadmap for closing gaps in care, research, and policy. Lancet. 2017; 390(10105): 1888-17.

3. Brasil. Ministério do Planejamento, Orçamento e Gestão. Instituto Brasileiro de Geografia e Estatística. Diretoria de Pesquisa. Coordenação de Trabalho e Rendimento. Pesquisa Nacional de Saúde 2013: percepção do estado de saúde, estilos de vida e doenças crônicas: Brasil, grandes regiões e unidades da federação. Rio de Janeiro: IBGE; 2014 [Internet] (Citado em 2 de fevereiro de 2018). Disponível em: https://www.ibge.gov.br/estatisticas/sociais/saude/9160-pesquisa-nacional-desaude.html?edicao $=9161 \& \mathrm{t}=$ downloads

4. Said S, Hernandez GT. The link between chronic kidney disease and cardiovascular disease. J Nephropathol. 2014; 3 (1): 99-104.

5. 5 World Health Organization. Global action plan for the prevention and control of noncommunicable diseases 2013-2020. WHO: Geneva; 2013 [Internet] (Citado em 2 de fevereiro de 2018). Disponível em: https://apps.who.int/iris/bitstream/handle/10665/94384/9789241506236eng.pdf;jsessionid=2E946049E8C96C 9BDE14365293F46F63? sequence=1

6. Hauschild DB, Schieferdecke ME, Leite CM, Nascimento MM. Composição corporal de pacientes com doença renal crônica em tratamento conservador. Rev. Med. UFPR. 2014; 1(2):47-53. 
7. Freiberg CK, Rossi L, Ceramico DCO. Antropometria e composição corporal. In: Rossi L, Caruso L, Galante, AP. Avaliação nutricional: novas perpectivas. 2a ed. Rio de Janeiro: Guanabara Koogan; 2015 p.171-174. ISBN: 978$85-277-2771-6$

8. Pitanga FJG, Lessa I. Razão cintura-estatura como discriminador do risco coronariano de adultos. Rev Assoc Med Bras. 2006; 52(3): 157-61.

9. Sampaio LR, Simões EJ, Assis AMO, Ramos LR. Validity ang reliability of sagittal abdominal diameter as a predictor of visceral abdominal fat. Arq Bras Endocrinol Metab. 2007; 51(6): 980-86.

10. Pitanga FJG, Lessa I. Sensibilidade e especificidade do índice de conicidade como discriminador do risco coronariano de adultos em Salvador, Brasil. Rev Bras Epidemiol. 2004; 7(3): 259-69.

11. Ben-Noun L, Sohar E, Laor A. A neck circumference as simple screening measure for identifying overweight and obese patients. Obesity Resarch. 2001; 9(8): 470-77.

12. Levey AS, Stevens LA, Schmid CH, et al. for the CKD-EPI (Chronic Kidney Disease Epidemiology Collaboration). A New Equation to Estimate Glomerular Filtration Rate. Ann Intern Med. 2009; 150 (5):604-612.

13. World Health Organization. Obesity: preventing and managing the global epidemic: report of a WHO Consultation on Obesity, Geneva, 3-5 June 1997. WHO: Geneva; 1998 [Internet]. (Acesso em 2 de fevereiro de 2018). Disponível em: http:// apps.who.int/iris/handle/10665/63854

14. National Cholesterol Education Program. Third report of the National Cholesterol Education Program (NCEP) Expert Panel on Detection, Evaluation, and Treatment of High Blood Cholesterol in Adults (Adult Treatment Panel III): Final Report. Circulation. 2002; 106(25): 3143-278

15. Valdez R. A simple model based index of abdominal adiposity. J Clin Epidemiol.1991; 44 (9): 955-6

16. Brasil. Ministério do Planejamento, Orçamento e Gestão. Instituto Brasileiro de Geografia e Estatística. Pesquisa Nacional de Saúde 2013: ciclos da vida: Brasil e grandes regiões. Rio de Janeiro: IBGE; 2015 [Internet] (Citado em 7 de agosto de 2020). Disponível em: https://biblioteca.ibge.gov.br/index.php/bibliotecacatalogo?view=detalhes\&id=294525

17. Brasil. Ministério da Saúde. Secretaria de Vigilância em Saúde. Departamento de Análise em Saúde e Vigilância de Doenças Não Transmissíveis. Vigitel Brasil 2019: vigilância de fatores de risco e proteção para doenças crônicas por inquérito telefônico: estimativas sobre frequência e distribuição sociodemográfica de fatores de risco e proteção para doenças crônicas nas capitais dos 26 estados brasileiros e no Distrito Federal em 2019. Brasília: Ministério da Saúde; 2020 [Internet] (Citado em 7 de agosto de 2020). Disponível em: https://portalarquivos.saude.gov.br/images/pdf/2020/April/27/vigitel-brasil-2019-vigilancia-fatores-risco.pdf 
18. Malta DC, Santos MAS, Andrade SSCA, Oliveira TP, Stopa SR, Oliveira MM, Jaime P. Tendência temporal dos indicadores de excesso de peso em adultos nas capitais brasileiras, 2006-2013. Cien Saúde Colet. 2016; 21(4): 1061-69.

19. Pereira IFS, Spyrides MHC, Andrade LMB. Estado nutricional de idosos no Brasil: uma abordagem multinível. Cad. Saúde Pública. 2016; 32(5): e00178814

20. Kamimura M A; Nerbass F B. Avaliação nutricional na doença renal crônica: o protagonismo da mensuração longitudinal. J Bras Nefrol. 2020; 42 (1): 4-5.

21. Silva DR. Obesidade: o verso e o reverso. J Bras Nefrol 2017; 39(3): 232-33

22. Kalantar-Zadeh K, Rhee CM, Chou J, et al. The obesity paradox in kidney disease: how to reconcile it with obesity management. Kidney Int Rep. 2017; (2):271-281.

23. Serra MM, Pereira LCO, Fontenele DF, Viveiros MTM, Lima RA. Condições clínicas e antropométricas de hipertensos atendidos em um centro de saúde de São Luís, MA. Rev Pesq Saúde. 2015; 16(2): 107-11.

24. Pinho CPS, Diniz ADS, Arruda IKG, Leite APDL, Petribú MMV, Rodrigues IG. Predictive models for estimating visceral fat: The contribution from anthropometric parameters. Plos One. 2017; 12(7): e0178958.

25. Monteiro CA, Cannon G, Lawrence M, Costa Louzada ML, Pereira Machado P. Ultra-processed Foods, Diet Quality, and Health Using the NOVA Classification System. Rome, Italy: FAO; 2019.

26. Roriz ANC, Passos LCS, Oliveira CC, Eickemberg M, Moreira PA, Sampaio LR. Evaluation of accuracy of anthropometric clinical indicators of visceral fat in adults and elderly. Plos One.2014; 9(12): e116449.

27. Kanh HS, Rissanen H, Bullard KM, Knekt P. The population distribuition of the saggittal abdominal diameter (SAD) and SAD/height ratio among Finnish adults. Clin Obes. 2014; 4(6): 333-38.

28. Strasser B, Volaklis K, Fuchs D, Burtscher M. Role of dietary protein and muscular fitness on longevity and aging . Aging Dis. 2018; 9 (1): 119-32.

29. World Health Organization. Who report on the global tobacco epidemic, 2019: offer help to quit tobacco use. WHO: Geneva; 2019 [Internet] (Citado em 15 de novembro de 2019). Disponível em: https://apps.who.int/iris/bitstream/handle/10665/326043/9789241516204-eng.pdf

30. World Health Organization. Global status report on alcohol and health 2014. WHO: Geneva; 2014 (Acesso em 5 de fevereiro de 2018). Disponível em :https://apps.who.int/iris/bitstream/handle/10665/112736/9789240692763_eng.pdf;jsessionid=8DA16C17EFD 5EDA3A56B99B47BA34D5D?sequence=1

31. Bezerra IN, Alencar SN. Associação entre excesso de peso e tamanho das porções de bebidas consumidas no Brasil. Rev. Saúde Pública. 2018; 52 (21): 1-11. 
32. Sociedade Brasileira de Cardiologia. Sociedade Brasileira de Hipertensão. Sociedade Brasileira de Nefrologia. $7^{a}$ Diretriz Brasileira de Hipertensão. Rev. Bras. Hipertens. 2017; 24(1): 1-91

33. Amorim RG, Guedes GS, Vasconcelos SML, Santos JCF. Doença renal do diabetes: cross-linking entre hiperglicemia, desequilíbrio redox e inflamação. Arq Bras Cardiol. 2019, 112 (5): 577-587.

34. Sociedade Brasileira de Diabetes. Diretrizes da Sociedade Brasileira de Diabetes 2019-2020. Clannad: São Paulo; 2019. [Internet] (Acesso em 10 de janeiro de 2020). Disponível em: https://www.diabetes.org.br/profissionais/images/DIRETRIZES-COMPLETA-2019-2020.pdf

\section{Colaboradoras}

Dutra AS contribuiu com a concepção e desenho do estudo, coleta de dados, análise e interpretação dos dados, redação e revisão crítica do artigo e da aprovação da versão final; Muniz GAS e Brito ACD contribuíram com a coleta de dados, análise e interpretação dos resultados e da redação e revisão crítica do artigo; Fontenele AMM contribuiu com a concepção e desenho do estudo; análise e interpretação dos dados, redação e revisão crítica do artigo; Conceição SIO contribuiu com a concepção e desenho do estudo; análise e interpretação dos dados, redação, revisão crítica e aprovação da versão final do artigo..

Conflito de Interesses: As autoras declaram não haver conflito de interesses

Recebido: 17 de maio de 2021

Aceito: 15 de outubro de 2021 